\title{
FUNDAMENTACIÓN FICHTEANA DE LA ÉTICA Y EL PROBLEMA DEL ALTRUISMO (THOMAS NAGEL)*
}

\author{
Jürgen STOLZENBERG (HALlE)
}

\begin{abstract}
Este artículo confronta la fundamentación fichteana de la filosofía práctica con la ética del altruismo de Thomas Nagel. El esbozo que hace Nagel de una ética del altruismo parte de la evidencia que tiene una persona de ser sólo una entre otras, y de ahí deduce la exigencia de hacer el bien a los otros, sin poner en juego intereses propios. Este punto de vista universal presupone la decisión de orientarse conforme a normas universales. Esta decisión encuentra fundamentación suficiente en la teoría fichteana de la voluntad pura. En efecto, sólo cuando una persona somere su acción a una voluntad pura suprapersonal, obtiene una conciencia de su libertad y su autodeterminación, que le permite orientarse conforme a normas universales. El concepto fichteano de una voluntad pura aparece por ello como un complemento necesario para la ética del altruismo de Nagel.
\end{abstract}

Con estas reflexiones quisiera presentar a Fichte como un compañero de diálogo de un filósofo contemporáneo. Ese filósofo es Thomas Nagel en sus reflexiones sobre la posibilidad del altruismo ${ }^{1}$. Hay buenas razones para ello.

* Conferencia pronunciada en la Facultad de Filosofía de la Universidad Complutense el 6 de noviembre de 2006 en el marco de la Jornada filosófica "Libertad, subjetividad y altruismo (a propósito de la Ética de Fichte)". Texto traducido del alemán por Jacinto Rivera de Rosales. Se trata de una versión modificada del artículo «Fichtes Willenslehre» publicado en el libro Fichtes praktische Philosophie. Eine systematische Einfübrung, ed. Hans Georg von Manz und Günter Zöller, Georg Olms Verlag: Reihe Europaea Memoria, Hildesheim u.a. 2006, pp. 93-110.

Me refiero en lo sucesivo a los libros de Thomas Nagel: The Possibility of Altruism, Oxford 1970, Princeton $1978^{2}$, citado como: Nagel, Altruism, y The last Word, Oxford 1997, citado como: Nagel, Word. 
Las reflexiones de Nagel sobre la ética del altruismo se distinguen por un concepto metódico, claro y a la vez atractivo. Partiendo de un rasgo fundamental de la comprensión que la persona tiene de sí misma, Nagel busca fundamentar la posibilidad de una ética del altruismo en cuanto expresión de esta autocomprensión. Esa fundamentación permite que se la ponga en una relación, sistemáticamente fructífera, con la fundamentación fichteana de la ética. Más exactamente, es la forma en la que Nagel pone en juego el concepto de una autoconciencia práctico-moral y en la que busca fundar la posibilidad de una ética del altruismo. Éste es, junto con el carácter propedéutico, el fundamento sistemático que aboga a favor de poner en diálogo Nagel y Fichte entre sí. En la primera parte sigo por tanto el rumbo de las reflexiones de Nagel hasta un punto en el que, por así decir, se da pie a la aparición de Fichte, y su contribución al asunto está motivada. Esa contribución es el tema de la segunda parte.

\section{I}

La autocomprensión de una persona, de la que parten las reflexiones de Nagel, consiste en que ella se capta sólo como un individuo o como una persona entre una multiplicidad de otros individuos o personas ${ }^{2}$. Tal autocomprensión, como diría Fichte, la aceptan todos sin necesidad de reflexión alguna sobre ello, y ésta es también la opinión de Nagel. Se mostrará que ella, tan evidente como parece, no carece de problemas conceptuales.

Por «altruismo" entiende Thomas Nagel una actitud o un comportamiento del ser humano que únicamente está dirigido por el convencimiento de que se debe hacer el bien al otro y evitar su daño. Aquí no se incluyen todas esas formas de noble autoinmolación por el bien de los otros, que es lo que comúnmente se llama altruis$\mathrm{mo}^{3}$. Aquí se piensa más bien en la disponibilidad «de actuar en consideración a los intereses de otra persona, sin necesidad de ulteriores motivos" ${ }^{4}$. Una tal actitud tiene su fundamento, según Nagel, en la autocomprensión de una persona, que se entiende a sí misma meramente como una más entre otras personas. Por qué esto es así y cómo hay que entenderlo, Nagel asegura poder mostrárnoslo.

\footnotetext{
2 Vgl. Nagel, Altruism, 16.

${ }^{3}$ Ebd.

${ }^{4}$ Nagel, Altruism, 79.
} 
A este respecto, Nagel introduce una distinción, que es fundamental para el desarrollo posterior de la argumentación ${ }^{5}$. La descrita autocomprensión de una persona puede ser entendida, según Nagel, como una unión de dos aspectos, perspectivas o puntos de vista, bajo los cuales una persona puede contemplarse a sí misma y al mundo: por una parte, el punto de vista personal, por otra, el punto de vista impersonal. El punto de vista personal es el de la primera persona. Aquí la persona misma es el sujeto o el autor de los juicios, convicciones y actitudes; ella es el lugar o el punto de vista desde el cual se refiere a sí misma y al mundo. De ese punto de vista propio se aparta el punto de vista impersonal; en él la persona se capta a sí misma y su lugar en el mundo exclusivamente entendiéndose como una persona entre otras, como "someone», como "cualquiera", no como "уо»".

El segundo paso describe una consecuencia de esta autocomprensión. Aquí Nagel llama la atención sobre el hecho de que la diferencia entre estas dos perspectivas no atañe al contenido proposicional de juicios y actitudes, sino sólo al modo de darse ese contenido ${ }^{7}$. Captarse a sí mismo como una persona más entre otras significa también captar a los otros del igual modo como personas, como a uno mismo. Esto significa que todos los contenidos que uno se puede atribuir desde el punto de vista personal (lo que se piensa, se siente, se opina o se tiene en mente), también se puede atribuir a los otros desde un punto de vista impersonal. La proposición personal "me alegro" no muestra por tanto, en relación con el contenido proposicional o el hecho de alegrarse, ninguna diferencia respecto al enunciado impersonal «él se alegra». La diferencia está únicamente en la perspectiva. Atañe sólo a la manera de captarlo, al acceso epistémico o incluso a la forma de darse ese contenido. De ello se sigue que una persona puede

${ }^{6}$ Cfr. Nagel, Altruism, 100 ss.

${ }^{6}$ Nagel, Altruism, 19. Véase al respecto también Thomas Nagel, The View from Nowhere, New York/Oxford, 1986, especialmente los capítulos IV u. VII - X. El primer comentario crítico, amplioy sistemático, procede de Dieter Henrich, Dimensionen und Defizite einer Theorie der Subjektivität en la revista Philosophische Rundschau, 36,1/2, Tübingen 1989. Véase también el comentario critico de Wilhelm Lütterfelds: Nagels „Blick von nirgendwo" - Eine aporetische Rehabilitierung der Transzendentalphilosophie?, en la revista Kant-Studien, 90, 1999/2, 204-222. En relación al concepto de Nagel de un yo objetivo y a sus reflexiones sobre idealismoy realismo, Lütterfelds reenvía al final de su comentario entre otros a la posición de Fichte $(220,222)$, sin aclararlo más pormenorizadamente.

${ }^{7}$ Cfr. Nagel, Altruism, 101. 
comprender enunciados que ella hace sobre sí misma, sus propias vivencias, convicciones, actitudes y acciones, como enunciados hechos en relación con una persona que en ese caso es idéntica a sí misma. Por consiguiente, quien se puede comprender bajo una perspectiva personal sólo en cuanto una persona entre otras, puede captarse también bajo una perspectiva impersonal. Puesto que dispone en esto a la vez de una conciencia de la diferencia de ambas perspectivas, está en la situación de decir que la persona a la que se refiere es ella misma.

Es fácil prever lo que de ahí se sigue para las condiciones de racionalidad de una teoría ética. Puesto que la autocomprensión de una persona se halla definida por medio de la unidad de esos dos puntos de vista o perspectivas, aquello que debe poder valer como principio ético en el sentido de criterio supremo de la moralidad de las acciones o de los propósitos que las dirigen, ha de estar de acuerdo con esta unidad. Eso significa que debe existir un tal principio univer$s a l^{\beta}$. Un principio ético es, en efecto, universal cuando es válido para todas las personas que se encuentren en una situación comparable, y cuando es aceptado por una persona sin consideración a su situación individual, es decir, cuando es aceptado de una manera impersonal. Con ello se cierra el primer círculo de la argumentación, pues justamente eso vale para el principio del altruismo, dado a conocer por Nagel. Ese principio descansa únicamente en el reconocimiento de la realidad y de los intereses de otras personas, sin tomar en consideración los intereses y los sentimientos del sujeto que actúa en cada caso. En consecuencia, a la base del altruismo se halla un principio universal, y por eso el altruismo puede ser entendido y fundado también como expresión de la unidad de las dos maneras descritas de autocomprensión de una persona.

\section{II}

El siguiente paso, el tercero, es decisivo. Con él sale a la luz aquella propiedad de la persona que he llamado al principio "autoconciencia práctico-moral». Aparece en las reflexiones de Nagel sobre el problema de la motivación. Ellas se plantean la cuestión sobre cómo la consideración de la realidad y de los intere-

${ }^{8}$ Cfr. Nagel, Altruism, 107. Cfr. también Th. Nagel: Universality and the Reflective Self, en: Christine Korsgaard: The Sources of Normativity, Cambridge 1996, $200-209$. 
ses de otras personas puede contener por sí misma un fundamento de motivación para una acción que se corresponda, sin que la persona tome en cuenta sus deseos, necesidades e inclinaciones individuales, que es lo que exige la posición del altruismo. La tesis de Nagel es que la respuesta a esta cuestión se desprende igualmente de la relación básica de la autocomprensión de una persona en cuanto una persona entre otras.

Tras los resultados hasta ahora obtenidos no parece sin embargo nada claro cómo ha de suceder esto. Dicho con más exactitud, no está claro cómo un fundamento de motivación en general puede ser deducido a partir de la unidad, acentuada por Nagel, entre las perspectivas personal e impersonal, las que definen la autocomprensión de una persona. Un fundamento de motivación - se le podría llamar así- ha de ser un fundamento válido para la persona y adecuado a ella, cuya acción está puesta en cuestión. Eso se sigue del concepto de un fundamento motivador de la acción. Tal fundamento parece que sólo puede ser comprendido a partir de una perspectiva personal, y no de una impersonal, pues sólo bajo aquella perspectiva la persona es capaz de contemplarse como sujeto y autora de sus acciones, y únicamente bajo esa perspectiva puede corresponderle a un fundamento una fuerza motivadora para la acción. De eso precisamente hace abstracción la perspectiva impersonal. Justamente lo que no debe jugar un papel substancial en la determinación del principio del altruismo y en la justificación de su validez, el punto de vista personal, parece que ahora tiene que jugar el papel fundamental en el problema de la motivación, si queremos ofrecer un fundamento que dirija la acción. No se ve cómo eso sea posible.

Este dilema lo busca solucionar Nagel con la siguiente consideración ${ }^{9}$. Parte de la idea de que un principio, cuyo contenido está subjetivamente, suprime la unidad en la autocomprensión de la persona como una persona entre otras, y eso a causa de que un principio tal excluye precisamente la consideración de los intereses de otras personas, gracias a la cual se define la autocomprensión de la persona. Por tanto no puede darse ningún principio subjetivamente condicionado en su contenido que sirva como fundamento de motivación. Luego sólo un principio objetivo, procedente de una perspectiva impersonal y que tiene que ser justificado, puede convertirse en un fundamento de motivación para el actuar

9 Véase Nagel, Altruism, 109-124, especialmente 122-123. 
de una persona. Pero eso sólo puede suceder, y éste es el paso decisivo en la reflexión de Nagel, cuando la persona emprende un cambio de perspectiva desde el punto de vista impersonal al personal, en el que el contenido del principio permanece a salvo. Este cambio de perspectiva consiste en que la persona convierte en cosa propia lo que ha reconocido como fundamento objetivo para la acción desde la perspectiva impersonal. Eso lo lleva a cabo decidiéndose a hacer realmente aquello que ha comprendido que debe ser hecho. El punto decisivo consiste en que la fuerza motivadora no es obtenida a partir de un principio subjetivo autosuficiente, sino exclusivamente del reconocimiento, realizado mediante la decisión de la persona, de un principio objetivo para su propio actuar. La perspectiva personal se convierte con ello, por así decir, en el lugar de la transformación, en el cual los fundamentos objetivos se convierten en motivos subjetivos de acción, y ese lugar de transformación lo posee la persona en su interior, la persona que uno es y que se comprende como tal.

A este cambio de perspectiva y a este fenómeno de la decisión le ha prestado Nagel una atención especial en sus últimas consideraciones acerca de la fundamentación de una ética: The last Word ${ }^{10}$. De aquí surge el tránsito a Fichte.

\section{III}

El interés de Nagel se dirige aquí sobre todo al convencimiento de que en una situación conflictiva la decisión que se ha de tomar, considerando todas las circunstancias relevantes, sobre lo que se debe hacer, y eso significa lo que es moralmente bueno, "queda a mi cargo", según lo expresa Nagel: "me toca a mí decidir, habiendo considerado todas las cosas, qué debo hacer»" ". Aquí se muestra, en efecto, la capacidad de una persona de distanciarse de todas las circunstancias personales y contingentes, y desde esa distancia tomar una decisión regida por criterios universalizables y aplicables universalmente. Justamente ése $-\mathrm{y}$ aquí Nagel introduce para su discusión en The last Word una idea nueva en relación con su primer libro- es el fundamento que permite concluir la realidad de la conciencia de la libertad:

${ }^{10}$ Cfr. Nagel, Word, 101ss.

11 Nagel, Word, 117. 
«El sentido de la libertad depende de que la decisión no haya sido tomada meramente desde mi punto de vista. Ésta es $[. .$.$] la exigencia de que mis$ acciones están conformes a normas universalmente aplicables» ${ }^{12}$.

Esa idea adquiere en este contexto un significado sistemático decisivo. Contiene implicaciones que apuntan más allá de lo que Nagel desarrolla al respecto. Permite, en primer lugar, ofrecer un fundamento epistémico para la unidad de las perspectivas personal e impersonal, que en la descripción de la autocomprensión de una persona fue sólo constatada por Nagel en sus primeras reflexiones y afirmada como un factum más allá del cual no se podía ir. Este fundamento no es, ciertamente, otro que el punto de vista personal. Su estructura lógica, sin embargo, ha de ser ahora ampliada en aspectos esenciales. Pues ahora se ha de decir que el punto de vista personal, bajo el cual una persona convierte un principio objetivo en la fundamentación de su propio actuar, no consiste únicamente en la conciencia de que se es esa persona, sino en que se dispone también de una conciencia de que uno mismo es el fundamento de la unidad de ambas perspectivas. En efecto, ese acto de decisión vive, por así decir, de que es realizado por la persona misma de una manera consciente; en consecuencia, no es sustituible por otros ni describible desde la perspectiva de otros. Al mismo tiempo, el contenido de ese acto, con el cual la persona se identifica, no está restringido únicamente a la persona que lo realiza, pues a ese contenido le sirve de base la idea de un principio universal, según el cual uno debe regirse en sus convicciones y acciones. Que ambas perspectivas de la persona que se es se mantienen, sólo puede hacerse consciente en la perspectiva personal. Por eso, la perspectiva personal es el fundamento de la conciencia de la unidad de las perspectivas personal e impersonal.

No obstante, la conciencia, actualizada en el acto personal, de la unidad de ambas perspectivas no es lo decisivo. La idea de un principio universal y su reconocimiento para el actuar propio es interpretada por Nagel como expresión de nuestra razón práctica. Su función la ve Nagel en que plantear «la exigencia de una justificación generalizable» - „the demand for generalizable justification " ${ }^{13}$. Esta función, según Nagel, se sigue inmediatamente de la autocomprensión descrita de una persona, y por tanto designa una característica esencial de nuestro

\footnotetext{
12 Nagel, Word, 117.

13 Nagel, Word, 109.
} 
ser persona. En efecto, quien se capta a sí exclusivamente como una persona entre otras y esto se convierte en la fundamentación de sus acciones, se comprende por ello, como se mostró al principio, a la vez como alguien que es capaz de reconocer principios universales, independientes de su propio punto de vista individual. Por eso, el punto de vista personal es también designado como el punto de vista de la razón práctica. Es el punto de vista bajo el cual una persona puede someter sus propios deseos, inclinaciones y convicciones a una exigencia de justificación capaz de ser universalizada, y puede actuar sobre ellos con esta exigencia.

Pero tampoco con ello son captados de un modo suficiente preciso ni el concepto ni la función sistemática de la perspectiva personal. En efecto, si se parte de que la función de la razón práctica y el reconocimiento de su fuerza normativa en relación con las propias acciones y los deseos, inclinaciones y convicciones que se hallan a la base, representan una característica esencial de nuestro ser persona, entonces se ha de decir que yo ahí reconozco algo como procedente de mí mismo en la medida en que me entiendo como persona. Por tanto, con el acto personal de reconocer un principio práctico universal logro también la conciencia de que la justificación universalizable, la que yo puedo hacer mía en referencia a mis propósitos de acción, no es otra cosa que la expresión de mi propia esencia como persona. Entonces la comprensión adecuada de ese acto personal no es la de captarlo como un acto de decisión meramente psicológico o como un acto simplemente lógico de aplicación de principios universales a la acción propia. Es, además de eso, expresión de una llamada autoconciencia originariamente práctica por parte de la persona que lo realiza, pues lo que la persona se apropia y aquello con lo que se identifica cuando hace suyo un principio práctico universal y deja que sea eficiente para su acción, eso no es otra cosa que la característica esencial de su ser persona, que ella actualiza y se atribuye. Luego tal autoconciencia originariamente práctica es propiamente aquello que constituye el carácter fundamental de la autocomprensión de una persona, el "núcleo interno de la persona» - "a core» ${ }^{14}$, como dice Nagel. Este paso, totalmente decisivo para una teoría de la persona y su papel en la fundamentación de una ética del altruismo, no lo ha dado Nagel.

14 Nagel, Altruism, 23. 
Algo parecido vale para el punto de vista de la decisión y la conciencia, allí implícita, de la libertad. Visto con exactitud, no es adecuado describirlo como tránsito desde una perspectiva impersonal a una personal, tal y como propone Nagel, sino más bien como actualización de la unidad, o más exactamente de la conciencia de unidad de ambas perspectivas. Esa unidad ha de ser ahora descrita con más precisión, a saber, la persona que posee este punto de vista no se refiere a sí en el mero sentido de que revierta sobre sí y haga suya una justificación universal de lo que debe ser hecho, sino en un sentido triplemente diferenciado, a saber, ella se entiende a sí como autora de esta exigencia que constituye el carácter esencial de su ser persona, en segundo lugar como receptora de la misma, y en tercer lugar como la instancia donde se lleva a cabo la apropiación y su realización. Ésa es la libertad en la que piensa Nagel, a saber, la libertad como autonomía, y ella se expresa en aquella autoconciencia originariamente práctica de una persona.

\section{IV}

No otra cosa era la visión originaria de Fichte. No la doctrina de un Yo puro, sino la de una conciencia originariamente práctica es la que está al inicio del camino filosófico de Fichte, y es también el fundamento de la teoría fichteana de la subjetividad concreta ${ }^{15}$. Si se intenta poner la posición de Fichte en una fructífera relación sistemática con lo dicho hasta ahora, nos hemos de dirigir al concepto fichteano de una voluntad pura. Desde él se puede captar con más precisión la teoría de Nagel de la autocomprensión de una persona y su función en la fundamentación de una ética del altruismo, y conducirlo a una forma exigida por el asunto mismo, pero que no ha sido desarrollada por Nagel.

15 Véase sobre esto Stolzenberg: Fichtes Begriff des praktischen Selbstbewußtseins. en: Fichtes Wissenschaftslehre 1794, ed. Wolfram Hogrebe, Frankfurt a. M. 1995, 71-95, y Reiner Wille. Ein Grundbegriff der Philosophie Fichtes, en: Revue Internationale de Philosophie, 206, 1998, 617-639 así como en Günter Zöller: Bestimmung zur Selbstbestimmung. Fichtes Theorie des Willens. en: Fichte-Studien, Bd. 7, Subjektivität, 101-118. Christian Klotz ha emprendido recientemente el ensayo de presentar la teoría fichteana de la subjetividad concreta y hacerla fructífera para la actual discusión sobre el concepto de la persona y de una conciencia volitivo-práctica de identidad de las personas. Cfr. Christin Klotz: Selbstbewusstsein und praktische Identität. Eine Untersuchung über Fichtes Wissenschaftslehre nova methodo, Frankfurt a. M. 2002. 
Si se quiere confrontar directamente la concepción de Nagel con la posición de Fichte, tiene pleno sentido partir de la explicación central, que acabamos de citar, de Nagel acerca del concepto de la libertad de la voluntad, que es por ahora la "última palabra» de Nagel sobre este asunto. La vuelvo a transcribir en su contexto:

«El sentido de la libertad depende de que la decisión no está tomada meramente desde $m i$ punto de vista. No se trata justamente de hallar las implicaciones de mi propia perspectiva, sino de la exigencia de que mis acciones estén conformadas a normas universalmente aplicables, que las convierte potencialmente en parte de un sistema armónico colectivo. De ese modo encuentro en mí las normas universales que me posibilitan salir de mí mismon ${ }^{16}$.

En la aclaración de Nagel están contenidos los siguientes supuestos. El primero consiste en que la capacidad de tomar una decisión sobre lo que se debe hacer implica tener una voluntad, pues querer algo significa empeñarse seriamente en hacer que sea real el objeto o estado de cosas pretendido. En caso contrario se podría haber hablado únicamente de la presencia de un mero deseo. Tener una voluntad significa, por tanto, que una persona se comprende a sí misma como autora de sus acciones, con las cuales se esfuerza por realizar, en el mundo en el que vive, determinados propósitos que ella denomina como propios. Atendiendo a la explicación anterior, cabe entender la tesis de Nagel como una tesis sobre la libertad de la voluntad en cuanto autonomía, y esta autonomía consiste en que una persona es capaz de decidirse a hacer abstracción de fundamentos de acción contingentes y de impronta individual, o distanciarse de ellos y someter desde sí misma su voluntad a fundamentos o patrones universales y universalmente aplicables, gracias a los cuales se convierten en elementos de un sistema coherente de acciones y de normas de acción.

La tesis de Fichte es que una persona que hace eso lleva a cabo algo más de lo que dice Nagel, algo decisivo. Este «algo más» es un presupuesto necesario. Consiste en que tal persona somete sus objetivos a lo que Fichte denomina una razón pura. Otra tesis de Fichte es que, sólo cuando se ha clarificado esto, pue-

16 Nagel, Word, 117. 
de verse realmente aquel "núcleo interno de la persona", como lo llama Nagel. Sólo el núcleo, así captado, de una persona define la conciencia de la autonomía de la que ella dispone, según Nagel, y sólo a partir de él se logra explicar la posibilidad de la decisión sobre alternativas concretas de acción. La tesis de Fichte puede ser entendida, por consiguiente, como una tesis crítica teórica tanto sobre los principios como sobre la subjetividad. Reclama un principio que ha de ser puesto en el fundamento de las reflexiones de Nagel sobre la posibilidad de una ética del altruismo, $y$ tomarla o no en consideración tiene consecuencias para la teoría de la persona que está a la base del esbozo de ética de Nagel.

Se ha de preguntar qué fundamentos ha dado a conocer Fichte al respecto, y si son fundamentos convincentes que han de ser aceptados por Nagel. Tendrían que ser aceptados por él en caso de que puedan ser deducidos a partir del concepto de una persona, propagado por él, y de su autocomprensión como un ser libremente actuante. Ése es de hecho el caso, y esto se puede mostrar partiendo del concepto fichteano de una voluntad pura.

El discurso de Fichte sobre una voluntad pura no apunta a una qualitas oculta separada. Se refiere a una idea de todos conocida y aceptada también por Nagel, a una idea que, como Nagel lo formula intuitivamente, vive en el estrecho pero profundo hueco entre nuestros deseos y nuestras acciones, a la idea de que podemos colocar nuestras acciones bajo la exigencia de una justificación capaz de ser universalizada ${ }^{17}$. El contenido normativo de esa idea puede ser captado claramente con independencia de los momentos empíricos y contingentes, y también de manera independiente a sus instantes concretos. Esto es posible porque la idea consiste en la exigencia invariable de su aplicación a todos los casos conforme a la realidad de una normatividad universal. Por eso puede ser denominada a priori. Dado que ella no se refiere a un estado de cosas teórico, sino a un contexto de acciones por medio del cual ha de ser realizada, esa idea tiene relación con la competencia práctica de una persona, es decir, con su voluntad. Entonces, a la voluntad de una persona se le ha de atribuir un modo de funcionar que puede ser descrito con el concepto de una voluntad pura. Con ello se piensa que la voluntad no está primariamente determinada por contenidos empíricos que hayan de ser realizados, sino únicamente por la forma lógica de una normatividad uni-

${ }^{17}$ Nagel, Word, 109/160. 
versal. Una persona que se sitúa en ese huevo entre sus deseos y lo que debe ser realizado, que hace suya la exigencia de una justificación universalizable de sus objetivos de acción, y que se esfuerza asimismo en cumplir esa exigencia, tal persona, se puede decir, se atribuye una voluntad pura. Ésta representa la condición necesaria bajo la cual la persona puede reconocer una justificación universalizable de su actuar. Eso ha de aceptarlo Thomas Nagel, y no sólo eso.

Una persona que se atribuye de esta manera una voluntad pura se determina a sí misma. Esto necesita una explicación. La autodeterminación de la que aquí se habla ocurre en el sentido específico de atribuirse un predicado descriptivo bajo una perspectiva final, pues el acto de autoatribución de una voluntad pura incluye analíticamente la disposición, mejor aún, la determinación de realizar una normatividad universal. En caso contrario, como se ha dicho antes, se trata de un deseo, de un apetito o de una esperanza, pero no de una voluntad. Podemos decir con Nagel que una persona que haga esto se ha distanciado ya, por así decir, de sus intenciones prácticas individuales y empírico-contingentes, y se ha considerado bajo una perspectiva universal. Por eso tiene sentido afirmar que sólo una persona que se atribuye una voluntad pura se constituye como persona en la unidad, descrita por Nagel, de las perspectivas personal e impersonal. Éste es, por lo demás, el presupuesto para que una persona sea capaz de configurar la conciencia de una identidad en relación con una secuencia de proyectos de acción ${ }^{18}$. Se puede decir, por consiguiente, que atribuirse una voluntad pura significa atribuirse el predicado real, lógicamente primero, gracias al cual una persona se constituye como tal. Ése es también el sentido de la conocida proposición inicial en la Ética de Fichte: "Yo sólo me encuentro a mí mismo en cuanto mí mismo queriendon ${ }^{19}$. El concepto de querer, que aquí utiliza Fichte, no hace referencia al sentido primario, común, de una intencionalidad siempre ocupada con la realidad de objetos concretos de una persona, sino al momento subjetivo distinto de aquél de la autodeterminación, en la que la persona se pone bajo el principio de una normatividad universal para su acción, una normatividad que Fichte entiende como expresión de la función práctica de la razón y que consti-

${ }^{18}$ Esta tesis sería aplicable ya a las primeras reflexiones de Nagel sobre la posibilidad de una identidad diacrónica de una persona. Cfr. Nagel, Altruism, 57ss.

${ }^{19}$ J. G. Fichte: Ética, Akal, Madrid, p. 84, en: J. G. Fichte-Gesamtausgabe der Bayerischen Akademie der Wissenschaften, hg. v. Reinhard Lauth u. Hans Jacob, Stuttgart-Bad Cannstatt 1962f., Bd. I/5, 37. 
tuye a la vez la esencia de una persona. Esa autoconciencia práctica es en consecuencia la expresión originaria de la autonomía de una persona, y no la capacidad de decidirse de manera universalmente válida bajo circunstancias empíricamente dadas, aunque esa autonomía sólo pueda ser realizada bajo dichas circunstancias ${ }^{20}$. También eso ha de aceptarlo Thomas Nagel, pues se sigue igualmente de su concepto de persona.

A ello se liga otro punto decisivo. Debido a la incondicionalidad que se ha de pensar en el concepto de una voluntad pura, su autoridad normativa no puede ser deducida de otro principio. Eso significa que la persona que reconoce la autoridad de una voluntad pura para su acción, une a ello la conciencia de estar destinado de una manera originaria a la autodeterminación. Esta persona, puede decirse de una manera aparentemente paradójica, descubre que no se ha decidido a sí misma a determinarse. De esto da cuenta Fichte interpretando esa relación consigo mismo como la conciencia de un "deber absoluto en cuanto exigencia categórica» ${ }^{21}$. Con ello no se piensa en el carácter de imperativo de la ley moral kantiana. La exigencia categórica fichteana no se dirige en primera línea, como sí sucede con la ley moral kantiana, a una voluntad empíricamente determina$\mathrm{da}$, sino que expresa únicamente la manera en la que aquella voluntad pura aparece al sujeto. Aparece de manera que la persona se experimenta como un estar determinado a la autodeterminación, acerca de lo cual no tiene alternativa ni es algo que pueda ser superado, y justamente en eso tiene la conciencia de su autonomía. Se puede decir por tanto que la conciencia que una persona tiene de su autonomía es la conciencia inmediata de encontrarse determinado a la autodeterminación sin alternativa posible.

Parece que Thomas Nagel tiene ante sí justamente esa idea, si bien no en la comprensión conceptual que se acaba de proponer, cuando escribe, ya en The Possibility of Altruism, con referencia a aquel mencionado «núcleo interno de la persona»:

${ }^{20}$ Cfr. Stolzenberg, Das Selbstbewusstsein einer reinen praktischen Vernunft. Zu den Grundlagen von Kants und Fichtes Theorien des sittlichen Bewusstseins, en: Metaphysik nach Kant? Stuttgarter Hegelkongreß 1987, hg. v. Dieter Henrich u. Rolf-Peter Horstmann, Stuttgart 1988,181208, especialmente 189-190.

21 J. G. Fichte: Wissenschaftslehre nova methodo, Kollegnachschrift Karl Christian Friedrich Krause, 1798/99, ed. Erich Fuchs, Hamburg 1982, 142, en lo sucesivo citada como: Fichte, WLnm, más el número de página. 
"No hay nada de lamentable en encontrarse uno mismo, en último término, ante algo frente a lo cual no podemos escoger el aceptarlo o rechazarlo. Aquello con lo que nos encontramos es probablemente uno mismo, un núcleo en el que no puede haber en modo alguno una elección por parte de la persona. Estas restricciones no elegidas en la elección se dan entre las condiciones de su posibilidad ${ }^{22}$.

Lo que muy probablemente quiere decir Nagel, aunque no lo dice, es que aquello frente a lo cual estamos y ante lo cual carecemos de elección es el carácter de la libertad misma, que de todos modos, siguiendo la concepción de Fichte, aparece en la forma de una ley a priori incondicionada, según la cual la persona se encuentra determinada a la autodeterminación. Justo ésta es de forma patente la relación básica que se da con la conciencia de la función de aquella razón práctica, de la que habla Nagel en The last Word. Ésa es justo la autoconciencia originariamente práctica de la que se hablaba al final del análisis de la teoría de Nagel, gracias a la cual una persona se constituye. Valga aquí la indicación de que el joven Schelling se adhirió a esta concepción con referencia explícita y elogiosa a Fichte. También para Schelling, tal voluntad pura es el origen y la "fuente" ${ }^{23}$ de la autoconciencia y a la vez el fundamento de una teoría de la subjetividad concreta. Sería interesante escuchar lo que Nagel tendría que decir al respecto.

En todo caso debería quedar claro que la teoría fichteana de una autoconciencia práctica pura tendría que hacerse sistemáticamente fructífera para las reflexiones de Nagel acerca del concepto de una persona más allá de lo dicho por él. Ella permite proporcionar un fundamento teórico en la subjetividad al concepto de Nagel acerca de la persona. Esto es exigido por el asunto mismo, pero Nagel no lo ha elaborado.

22 Nagel, Altruism, 23.

${ }^{23}$ Cfr. F. W. J. Schelling: Allgemeine Übersicht der neuesten philosophischen Literatur, en: AA I/4, 128, así como System des transzendentalen Idealismus, en: SW III, 535. Sobre este asunto véase también Stolzenberg, Autonomie. Zu Schellings Begründung der praktischen Philosophie, en: System als Wirklichkeit. 220 Jahre Schellings "System des transzendentalen Idealismus", ed. Christian Danz, Claus Dierksmeier u. Christian Seysen (Kritisches Jahrbuch der Philosophie, Bd. 6) Würzburg 2001, 41-56. 


\section{$\mathbf{V}$}

Lo mismo vale para el principio del altruismo. La tesis de Nagel era que este principio puede ser fundamentado partiendo de la autocomprensión, descrita al inicio, de una persona, y el punto álgido de su argumentación al respecto era ver que el reconocimiento ilimitado requerido de la realidad de las otras personas sólo puede ser justificado cuando una persona es capaz de contemplarse a sí misma de una manera impersonal y hace suyos fundamentos objetivos. $\mathrm{Al}$ intentar Nagel en The last Word ligar su concepto de persona de una manera claramente más estrecha a la conciencia de la libertad en cuanto autonomía, y dar ese paso en continuidad con su primer proyecto ético, surgen de aquí también consecuencias para la interpretación del principio del altruismo, consecuencias que Nagel no ha sacado, pero que él hubiera podido extraer fácilmente.

En efecto, si se intenta ligar la idea de la autonomía con la exigencia del altruismo de reconocer la realidad de las otras personas sin tomar en consideración los propios deseos y necesidades, entonces se ha de observar primeramente que Nagel lo ha tenido en cuenta en la medida en que, enlazando con sus primeras reflexiones, atribuye a la persona de ahí en adelante un valor objetivo ${ }^{24}$. Esta tesis, por su parte, se desprende de la capacidad de una persona para determinarse a sí misma según fundamentos objetivos haciendo abstracción de preferencias contingentes y subjetivas. Bajo esta perspectiva, la exigencia de reconocer la realidad de otras personas se ha de entender de manera que este valor objetivo, que les corresponde originariamente, debe ser asegurado y garantizado. Pero con ello no queda clara la propia fuerza del argumento. Ésta se muestra sólo cuando se considera que ese valor objetivo encuentra su expresión en la conciencia de la libertad como autonomía y que el reconocimiento de la realidad de las otras personas por tanto no puede ser ni significar otra cosa que el reconocimiento y la garantía de esta autonomía suya originaria.

Eso es lo que Kant, al igual que Fichte, llaman la dignidad de la persona, que prohíbe considerar y utilizar a las personas como si fuesen cosas, o sea, como medios para fines cualesquiera. Las personas son más bien fines en sí mismas, en la medida en que el fundamento de su existencia como personas que actúan

${ }^{24}$ Nagel, Word, 123. 
moralmente lo tienen en sí mismas. Por consiguiente, les corresponde también un valor objetivo y absoluto. La defensa de Nagel en favor de una ética del altruismo conduce por ello a la defensa de una ética de la humanidad, que descansa en la comprensión que una persona tiene de sí misma en cuanto ser libre y autónomo.

Con este resultado hay que volver de nuevo a la doctrina de Fichte sobre la libertad. En efecto, se puede mostrar fácilmente que la ética del altruismo de Nagel, en el sentido de una ética de la autonomía y de la humanidad, puede ser reconstruida a partir del concepto fichteano de una voluntad pura, pues lo que Fichte denomina "reino de los seres racionales»" ${ }^{25}$, la famosa "síntesis del mundo de los espíritus", no es otra cosa que aquella esfera o dominio de la voluntad pura, en la cual esta voluntad aparece como la ley de todas las voluntades individuales. Por eso Fichte llama a la voluntad pura también «ley práctica» o "la ley del mundo suprasensible» ${ }^{26}$. Cuando Nagel habla de que las acciones individuales, que se conforman a normas universales, devienen "partes de un sistema colectivo armónico" - „parts of a harmonious collective system"-, entonces se ha de validar el principio, no nombrado por Nagel, de este sistema colectivo con el concepto fichteano de una voluntad pura. Pero con ello se ha hecho claro de nuevo que Fichte puede llegar a ser algo más que justificado para las demandas de nuestro presente.

25 Fichte, WLnm, 150 u. 152. 297.

${ }^{26}$ J. G. Fichte: Die Bestimmung des Menschen, en: Fichtes Werke, hg. v. I. H. Fichte, Bd. II, 